\title{
A COMPLEX THAT RESOLVES THE IDEAL OF MINORS HAVING $n$ - 1 COLUMNS IN COMMON
}

\author{
J. F. ANDRADE AND A. SIMIS
}

\begin{abstract}
Let $\boldsymbol{A}$ be an $n \times m$ matrix $(m>n)$ with entries in a noetherian ring $R$, let $J$ be the ideal of $R$ generated by the $n \times n$ minors that are formed with $n-1$ fixed columns of $A$. Necessary and sufficient conditions are given in order that a suitably defined complex be a free resolution of the $R$-module $R / J$. The complex is closely related to the complexes of Buchsbaum-Rim $[2,82]$ and it is perhaps worthwhile mentioning that a special case of the present result was obtained by Buchsbaum-Eisenbud in a different context [1, Theorem 8.1].
\end{abstract}

We will give the result in a basis-free manner. The following bit of notation will be required. Let $F \stackrel{f}{\rightarrow} G$ be a map of free $R$-modules with $\operatorname{rank} F=m>\operatorname{rank} G=$ $n$. By $I_{t}(f)$ is meant the ideal of $R$ generated by the $t \times t$ minors of a matrix representing $f$. If $t=\operatorname{rank} f$, we simply write $I(f)$ instead of $I_{t}(f)$. If $\Lambda_{-}^{i}$ and $S_{i}\left({ }_{-}\right)$ denote, respectively, the $i$ th exterior power and the $i$ th symmetric power of a module, then the complex of Buchsbaum-Rim is

$$
0 \rightarrow \Lambda^{m} F \otimes S_{m-n-1}\left(G^{*}\right) \stackrel{d_{m-n+1}}{\rightarrow} \cdots \stackrel{d_{3}}{\rightarrow} \Lambda^{n+1} F \otimes S_{0}\left(G^{*}\right) \stackrel{e}{\rightarrow} F \stackrel{f}{\rightarrow} G
$$

where $\varepsilon$ is a suitable composite:

$$
\Lambda^{n+1} F \otimes S_{0}\left(G^{*}\right) \stackrel{\sim}{\rightarrow} \Lambda^{n+1} F \otimes \Lambda^{n} G^{*} \stackrel{1 \otimes \Lambda^{n *}}{\rightarrow} \Lambda^{n+1} F \otimes \Lambda^{n} F^{*} \rightarrow F
$$

(cf. [2, §2] and $d_{i}, i \geqslant 3$, is defined as in the complex of Eagon-Northcott [ibid.] or [3]). We will make no use of the explicit description of these maps and, accordingly, give no details. We will however rely heavily on the main result about the above complex, namely, that it is a free resolution (of coker $f$ ) if and only if the depth of $I_{n}(f)$ is $>m-n+1[2$, Theorem 2.1].

Now, assume there is given a decomposition $F=F^{\prime} \oplus F^{\prime \prime}$, with $F^{\prime}$ and $F^{\prime \prime}$ free modules. Let $\Lambda^{n+1} F \otimes S_{0}\left(G^{*}\right) \stackrel{\varepsilon^{\prime \prime}}{\rightarrow} F^{\prime \prime}$ be the composite $\Lambda^{n+1} F \otimes S_{0}\left(G^{*}\right) \rightarrow F \stackrel{p}{\rightarrow} F^{\prime \prime}$, where $p$ is the natural projection. Also, denote by $f^{\prime}$ the restriction of $f$ to $F^{\prime}$. We then have:

LemMa. Assume that the map $f^{\prime}: F^{\prime} \rightarrow G$ is injective. Then the following are equivalent.

(i) The complex

$$
0 \rightarrow \Lambda^{m} F \otimes S_{m-n-1}\left(G^{*}\right) \stackrel{d_{m-n+1}}{\rightarrow} \cdots \stackrel{d_{3}}{\rightarrow} \Lambda^{n+1} F \otimes S_{0}\left(G^{*}\right) \stackrel{\varepsilon}{\rightarrow} F
$$

is a free resolution of $\operatorname{im}(f)$.

Received by the editors August 13, 1979 and, in revised form, December 20, 1979.

AMS (MOS) subject classifications (1970). Primary 13C10, 13D99; Secondary 18G10. 
(ii) The complex

$$
0 \rightarrow \Lambda^{m} F \otimes S_{m-n-1}\left(G^{*}\right) \stackrel{d_{m-n+1}}{\rightarrow} \cdots \stackrel{d_{3}}{\rightarrow} \Lambda^{n+1} F \otimes S_{0}\left(G^{*}\right) \stackrel{e^{\prime \prime}}{\rightarrow} F^{\prime \prime}
$$

is a free resolution of $\operatorname{im}(f) / \mathrm{im}\left(f^{\prime}\right)$.

Proof. This follows from the following commutative diagram with exact columns.

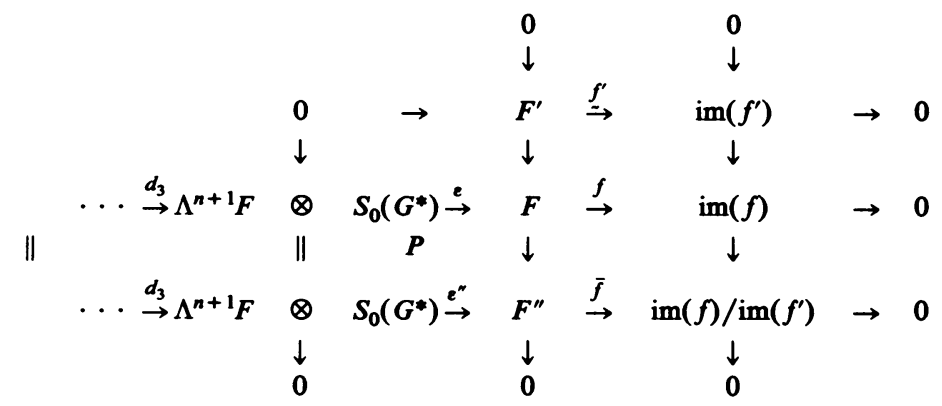

where $\bar{f}$ is naturally induced by $f$ via the identification $F^{\prime \prime} \simeq F / F^{\prime}$.

THEOREM. Let $F \stackrel{f}{\rightarrow} G$ be a map of free modules, with rank $F=m>\operatorname{rank} G=n$. Let there be given a decomposition $F=F^{\prime} \oplus F^{\prime \prime}$ into free modules, with rank $F^{\prime}=$ $n-1$. Set $f^{\prime}: F^{\prime} \rightarrow G$ for the restriction map. Then the following conditions are equivalent.

(i) depth $I_{n}(f) \geqslant m-n+1$ and depth $I_{n-1}\left(f^{\prime}\right)>2$.

(ii) The complex

$$
\begin{aligned}
& 0 \rightarrow \Lambda^{m} F \otimes S_{m-n-1}\left(G^{*}\right) \stackrel{d_{m-n+1}}{\rightarrow} \ldots \\
& \stackrel{d_{3}}{\rightarrow} \Lambda^{n+1} F \otimes S_{0}\left(G^{*}\right) \rightarrow F^{\prime \prime} \rightarrow \operatorname{im}(f) / \operatorname{im}\left(f^{\prime}\right) \rightarrow 0
\end{aligned}
$$

is exact and $\operatorname{im}(f) / \operatorname{im}\left(f^{\prime}\right) \simeq\left(\Lambda^{n} f\right)\left(F^{\prime \prime}\right)$ (where $F^{\prime \prime}$ sits naturally inside $\Lambda^{n} F=$ $\Lambda^{n}\left(F^{\prime} \oplus F^{\prime \prime}\right)=\bigoplus_{i=0}^{n}\left(\Lambda^{i} F^{\prime} \otimes \Lambda^{n-i} F^{\prime \prime}\right)$ as $\left.\Lambda^{n-1} F^{\prime} \otimes \Lambda^{1} F^{\prime \prime}\right)$.

REMARK. Fixing bases we get the result exactly as stated in the "abstract", as $\left(\Lambda^{n} f\right)\left(F^{\prime \prime}\right)$ will be thus identified with the ideal of $n \times n$ minors involving the columns corresponding to the submodule $F^{\prime}$.

Proof. (i) $\Rightarrow$ (ii). Since depth $I_{n}(f) \geqslant m-n+1$, the complex (1) is exact. On the other hand, $f^{\prime}$ is injective as depth $I_{n-1}\left(f^{\prime}\right)>1$. Therefore, by the lemma, we get the assertion that the above complex is exact. To prove the second assertion we will use the remaining assumption that depth $I_{n-1}\left(f^{\prime}\right)>2$. Namely, we have the exact sequence (Hilbert-Burch) $0 \rightarrow F^{\prime} \stackrel{f^{\prime}}{\rightarrow} G \stackrel{\varphi}{\rightarrow} R$ where $\varphi=\Lambda^{n-1}\left(f^{\prime}\right)^{*}$ (i.e., $\varphi(G)$ $\left.=I_{n-1}\left(f^{\prime}\right)\right)$. But, then

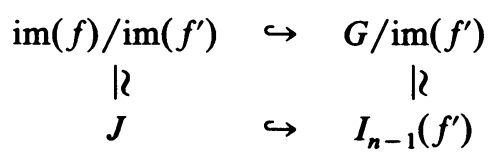

for some ideal $J$. We claim that $J=\left(\Lambda^{n} f\right)\left(F^{\prime \prime}\right)$. To see this, we fix a free basis $\left\{e_{k}\right\}$ of $G$ and generators $\left\{v_{l}\right\}$ of $\operatorname{im}(f)$. Writing $v_{l}=\sum_{k=1}^{n} \alpha_{k l} e_{k}, l=1, \ldots, m$, and 
applying to $v_{l}$ the composite $\operatorname{im}(f) \hookrightarrow G \stackrel{\varphi}{\rightarrow} R$, we get the element $\sum_{k=1}^{n} \alpha_{k l} D_{k}^{\prime}$, where $D_{k}^{\prime}(k=1, \ldots, n)$ are the $(n-1) \times(n-1)$ minors of $f^{\prime}$ (up to sign) in the basis $\left\{e_{k}\right\}$. By the Laplace expansion, $\sum_{k=1}^{n} \alpha_{k l} D_{k}^{\prime}$ is the $n \times n$ minor of $f$ formed with the $n-1$ columns corresponding to $\operatorname{im}\left(f^{\prime}\right)$ and the $l$ th column of $\left(\alpha_{k l}\right)$. Thus, we obtain the inclusion $J \subset\left(\Lambda^{n} f\right)\left(F^{\prime \prime}\right)$. The reverse inclusion is obtained in a similar way.

(ii) $\Rightarrow$ (i). The hypothesis is to the effect that

$$
\begin{aligned}
0 \rightarrow \Lambda^{m} F \otimes S_{m-n-1}\left(G^{*}\right) & \stackrel{d_{m-n+1}}{\rightarrow} \cdots \\
& \stackrel{d_{3}}{\rightarrow} \Lambda^{n+1} F \otimes S_{0}\left(G^{*}\right) \stackrel{e^{\prime \prime}}{\rightarrow} F^{\prime \prime} \stackrel{d_{1}}{\rightarrow} R \rightarrow R / J \rightarrow 0
\end{aligned}
$$

is exact, where $J=\left(\Lambda^{n} f\right)\left(F^{\prime \prime}\right)$. Then depth $J \geqslant 1$; hence depth $I_{n-1}\left(f^{\prime}\right)>1$ too as $J \subset I_{n-1}\left(f^{\prime}\right)$. Therefore, $f^{\prime}$ is injective and, by the lemma, the complex of Buchsbaum-Rim is exact. In other words, depth $I_{n}(f)>m-n+1$. Next, to see that depth $I_{n-1}\left(f^{\prime}\right)>2$ we show that actually depth $J>2$. Namely, in the "generic" case (i.e., the case of a matrix of distinct indeterminates $T_{k l}$ over $R$ ), the condition in (i) are clearly satisfied; hence, by the first part of the theorem, the corresponding generic complex is a resolution of the "generic" ideal $\tilde{J}$. But, clearly, depth $\tilde{J}>2$. Therefore, $\sqrt{I\left(\tilde{\varepsilon}^{\prime \prime}\right)} \subset \sqrt{I\left(\tilde{d}_{1}\right)}$ [1, Theorem 2.1(b)]. Specializing $T_{k l} \mapsto \alpha_{k l}$, we still get $\sqrt{I\left(\varepsilon^{\prime \prime}\right)} \subset \sqrt{I\left(d_{1}\right)}$. Since $(2)$ is assumed to be exact, depth $I\left(\varepsilon^{\prime \prime}\right)>2[1$, Theorem 1.2]. Therefore, depth $J=\operatorname{depth} I\left(d_{1}\right) \geqslant 2$.

Acknowledgements. We wish to heartily thank D. Eisenbud and the referee who suggested many improvements in the proof of the Theorem: our original proof was much lengthier and less elegant.

\section{REFERENCES}

1. D. Buchsbaum and D. Eisenbud, Some structure theorems for finite free resolutions, Advances in Math. 12 (1974), 84-139.

2. _ Remarks on ideals and resolutions, Symposia Mathematica, Vol. 11, Academic Press, London, 1973, pp. 191-204.

3. J. Eagon and D. Northcott, Ideals defined by matrices and a certain complex associated to them, Proc. Royal Soc. Ser. A 269 (1962), 188-204.

instituto de Matemática, Universidade federal da Bahia, Rua Caetano de Moura, 99Federacĩo, 40.000 Salvador, Bahia, Brasil

instituto de Matemática Pura e Aplicada (IMPA), Rua luis de Camões, 68-Centro, 20.060 Rio DE JANEIRO, R.J., BRASIL 